\title{
A Cubic Decomposition of Sequences of Orthogonal Polynomials on the Unit Circle
}

\author{
MANUEL ALFARO ${ }^{\mathrm{a}, *}$, MARÍA JOSÉ CANTERO ${ }^{\mathrm{b}, \dagger}$ and FRANCISCO MARCELLÁN ${ }^{\mathrm{c}, \neq}$ \\ ${ }^{a}$ Departamento de Matemáticas, Universidad de Zaragoza, 50009 Zaragoza, Spain; \\ ${ }^{\mathrm{b}}$ Departamento de Matemática Aplicada, Universidad de Zaragoza, 50015 Zaragoza, Spain; \\ ${ }^{\mathrm{C}}$ Departamento de Matemáticas, Universidad Carlos III de Madrid, Avenida de la Universidad 30, \\ 28911 Leganés, Madrid, Spain
}

\begin{abstract}
In this paper we will discuss the problem of generation of sequences of orthogonal polynomials with respect to measures supported on the unit circle from a given sequence of orthogonal polynomials using a perturbation of a cubic sieved process. The basic tools are the Szego forward recurrence relation as well as the fact of the coprimality of orthogonal polynomials on the unit circle and their corresponding reverse polynomials. We also give the connection between the associated orthogonality measures. Finally, some examples of this cubic decomposition are shown.
\end{abstract}

Keywords: Orthogonal polynomials; Reflection parameters; Carathéodory functions

(2000) AMS Subject Classification: $42 \mathrm{C} 05$

\section{INTRODUCTION}

The role of orthogonal polynomials on the unit circle in circuit and system theory is very well recognized. Orthogonal polynomials with respect to measures supported on the unit circle are directly related to problems of Fourier expansions, characterization of positive functions and stable polynomials, least-square polynomial approximations, and spectral operator analysis.

A set of orthogonal polynomials on the unit circle is classically associated with a Toeplitz matrix which has the structure of the covariance matrix of a discrete stationary stochastic process. The properties of orthogonal polynomials on the unit circle have

\footnotetext{
*Corresponding author. E mail: pacomarc@ing.uc3m.es

*E mail: alfaro@posta.unizar.es

${ }^{\dagger}$ E mail: mjcante@posta.unizar.es
} 
been extensively used in least square estimation problems although they appear in that context quite independently of the Szego theory, as a natural consequence of the pioneering work by Wiener, Kolmogorov and Krein. As an example, the Levinson algorithm, widely used by statisticians, is well known to be built on the forward recurrence relation that such orthogonal polynomials on the unit circle satisfy. The Schur-Cohn algorithm is well known to be described from the backward recurrence relation for our orthogonal polynomials on the unit circle (see [2]).

In the real line, there exists a very important specific set of orthogonal polynomials related to eigenfunctions of differential or difference operators, whose symmetrization factors are same very well known distributions. Beta, gamma and normal distributions for Jacobi, Laguerre and Hermite polynomials, respectively, in the continuous case. Poisson, binomial, Pascal and hypergeometric distributions for Charlier, Kravchuk, Meixner and Hahn polynomials, respectively, in the discrete case (see [14]).

For the unit circle there are very few explicit examples and they have not been used very often. One of the reasons is the fact that they enter into a different class of problems than the classical orthogonal polynomials on the real line do (see [14]). In such a sense, we have very powerful and theoretical tools to work but we need to realize a constructive theory in order to give an important improvement in the analysis of their properties. In the nineties a hard work has been achieved in such a direction taking into account some different approaches from the perturbation of measures of orthogonality ([11]), reflection parameters ([9]) or the polynomials themselves, $([3,6,7])$. The aim of the present contribution is to give some new examples, from the above point of view, related with a cubic decomposition of sequences of orthogonal polynomials on the unit circle. We continue the work started in [3] for the quadratic case.

The structure of this article is the following. First we give some basic definitions and results concerning orthogonal polynomials related to Hermitian linear functionals and we state our main result. In Section 2, we include its proof. Finally, in Section 3 we give some examples and, in particular, we obtain the explicit expression of the C-function. Thus in a positive definite case the absolutely continuous component of the orthogonality measure is obtained.

Let $u$ be a linear functional on the linear space of the Laurent polynomials $\Lambda=\operatorname{span}\left\{z^{k}: k \in \mathbb{Z}\right\}$ such that if $u_{n}=\left\langle u, z^{n}\right\rangle$, then $u_{n}=\left\langle u, z^{n}\right\rangle=\bar{u}_{n}, n \in(\mathbb{N} \cup\{0\})$.

Using this linear functional, we can introduce a bilinear form on $\mathbb{P}$, the linear space of polynomials with complex coefficients, in the following way

$$
\langle p, q\rangle=\left\langle u, p(z) \bar{q}\left(z^{1}\right)\right\rangle, \quad \forall p, q \in \mathbb{P} .
$$

Notice that in such a case, the shift operator is unitary with respect to the above bilinear form, which we will assume is quasi-definite, i.e., the principal submatrices $T_{n}, n \in \mathbb{N}$, of the Gram matrix $T$

$$
T=\left[\left\langle z^{i}, z^{j}\right\rangle\right]_{i, j=0}^{\infty}
$$

are nonsingular for every $n \in \mathbb{N}$. The infinite Gram matrix $T$ is a Toeplitz matrix because of the shift operator is a unitary operator with respect to the above inner product. If the principal submatrices $\left(T_{n}\right)$ are positive definite, then the linear functional $u$ is 
said to be positive definite. In such a case, there exists a unique positive Borel measure $\mu$ supported on $\mathbb{T}$ such that

$$
\langle u, p\rangle=\int_{\mathbb{T}} p(z) d \mu(z) .
$$

See $[2,5]$.

In such conditions, there exists a sequence of monic polynomials $\left(\Phi_{n}\right)$, such that

(i) $\operatorname{deg} \Phi_{n}=n$,

(ii) $\left\langle\Phi_{n}, \Phi_{m}\right\rangle=M_{n} \delta_{n, m}, M_{n} \neq 0$.

The sequence $\left(\Phi_{n}\right)$ defined as above is said to be a monic orthogonal polynomial sequence (MOPS) with respect to the linear functional $u$.

Furthermore, from elementary properties of the Toeplitz matrices we get $\left|\Phi_{n}(0)\right| \neq 1$. The values $\left(\Phi_{n}(0)\right)$ are called the reflection parameters for the linear functional $u$.

It is very well known that $\left(\Phi_{n}\right)$ satisfies a forward recurrence relation:

$$
\begin{aligned}
& \Phi_{n}(z)=z \Phi_{n} 1(z)+\Phi_{n}(0) \Phi_{n}^{*}{ }_{1}(z), \quad n=1,2, \ldots, \\
& \Phi_{0}(z)=1,
\end{aligned}
$$

as well as a backward recurrence relation:

$$
\begin{aligned}
& \Phi_{n}(z)=\left(1-\left|\Phi_{n}(0)\right|^{2}\right) z \Phi_{n}{ }_{1}(z)+\Phi_{n}(0) \Phi_{n}^{*}(z), \quad n=1,2, \ldots, \\
& \Phi_{0}(z)=1,
\end{aligned}
$$

which were obtained by G. Szegö (see [13]). By means of $\Phi_{n}^{*}(z)$ we denote the reversed polynomial of $\Phi_{n}$.

$$
\Phi_{n}^{*}(z)=z^{n} \bar{\Phi}_{n}\left(z^{1}\right) .
$$

Notice that the forward recurrence relation characterizes an MOPS with respect to a linear functional $u$ (Favard's Theorem) if and only if $\left|\Phi_{n}(0)\right| \neq 1$ (see [5]). For a proof of the positive definite case, i.e., $\left|\Phi_{n}(0)\right|<1$, see [4].

We can associate with the linear functional $u$ a formal series

$$
F(z):=u_{0}+2 \sum_{n=1}^{\infty} \bar{u}_{n} z^{n} .
$$

In the positive definite case, $F$ is an analytic function in the unit disk and $\operatorname{Re} F(z) \geq 0$. In the literature $F$ is said to be a Carathéodory function or a $\mathrm{C}$-function $($ see $[9,10])$.

Lemma 1 The zeros of the polynomial $\Phi_{n}$ do not lie on the unit circle.

Proof If $\Phi_{n}(\alpha)=0$ with $|\alpha|=1$, then

$$
\Phi_{n}^{*}(\alpha)=\alpha^{n} \overline{\Phi_{n}(\alpha)}=0 .
$$

Taking into account the backward recurrence relation, $\Phi_{n} 1_{1}(\alpha)=0$ and thus, by iteration, $\Phi_{0}(\alpha)=0$ which is a contradiction. 
Lemma 2 The polynomials $\Phi_{n}$ and $\Phi_{n}^{*}$ are relatively prime polynomials.

Proof See [1].

As a consequence, we get:

Proposition 3 If the polynomials $\Phi_{n}$ and $\Phi_{n}^{*}$ verify $p(z) \Phi_{n}(z)=q(z) \Phi_{n}^{*}(z)$ where $p$ and $q$ are polynomials with $\operatorname{deg} p \leq n-1, \operatorname{deg} q \leq n-1, n \geq 2$, then $p \equiv q \equiv 0$.

We are interested in the following problem.

Given a MOPS $\left(\Phi_{n}\right)$ with respect to a quasi-definite linear functional $u$, to find polynomials $A_{n, i}, B_{n, i}, C_{n, i}, i=1,2, n \in \mathbb{N}$ with degree less than or equal to $n$ such that the sequence $\left(\Psi_{n}\right)$ of monic polynomials given by

$$
\begin{aligned}
\Psi_{3 n}(z) & =\Phi_{n}\left(z^{3}\right)+z A_{n} \quad 1,1\left(z^{3}\right)+z^{2} A_{n} \quad 1,2\left(z^{3}\right), \quad n \geq 0 \\
\Psi_{3 n+1}(z) & =z \Phi_{n}\left(z^{3}\right)+B_{n, 1}\left(z^{3}\right)+z^{2} B_{n} \quad 1,2\left(z^{3}\right), \quad n \geq 0 \\
\Psi_{3 n+2}(z) & =z^{2} \Phi_{n}\left(z^{3}\right)+C_{n, 1}\left(z^{3}\right)+z C_{n, 2}\left(z^{3}\right), \quad n \geq 0
\end{aligned}
$$

with the convention $A_{1,1}=A_{1,2}=B_{1,2} \equiv 0$, is a MOPS with respect to a quasi-definite linear functional $v$.

Notice that

$$
\begin{gathered}
\Psi_{3 n}(0)=\Phi_{n}(0), \\
\Psi_{3 n+1}(0)=B_{n, 1}(0), \\
\Psi_{3 n+2}(0)=C_{n, 1}(0) .
\end{gathered}
$$

An analog question for the quadratic case has been already solved in [3]. There it has been proved that, if $\left(\Phi_{n}\right)$ is a MOPS then, the polynomials defined by

$$
\begin{aligned}
R_{2 n}(z) & =\Phi_{n}\left(z^{2}\right)+z B_{n} 1\left(z^{2}\right), \\
R_{2 n+1}(z) & =z \Phi_{n}\left(z^{2}\right)+D_{n}\left(z^{2}\right),
\end{aligned}
$$

are orthogonal with respect to a quasi-definite linear functional if and only if $D_{N}(0) \neq 0$ for at most one $N \in \mathbb{N}$ and the polynomials $B_{n}, D_{n}$ satisfy

(i) If $D_{n}(0)=0$ for every $n \in \mathbb{N}$, then $B_{n}(0)=D_{n}(0)=0$ for every $n \in \mathbb{N}$, i.e. $R_{2 n}(z)=\Phi_{n}\left(z^{2}\right), R_{2 n+1}(z)=z \Phi_{n}\left(z^{2}\right)$.

(ii) If $D_{N}(0) \neq 0$, then $B_{n}(z)=D_{n}(z)=0$ for $n=0,1,2, \ldots, N-1$ as well as

$$
\begin{aligned}
D_{N}(z) & =D_{N}(0) \Phi_{N}^{*}(z), \\
B_{N}(z) & =D_{N}(z)+\Phi_{N+1}(0) D_{N}^{*}(z), \\
D_{n}(z) & =z B_{n \quad 1}(z), \quad n \geq N+1, \\
B_{n+1}(z) & =z B_{n}(z)+\Phi_{n+2}(0) B_{n}^{*}(z), \quad n \geq N .
\end{aligned}
$$


Notice that in such a case, the reflection parameters $R_{n}(0)$ are given by

$$
\begin{aligned}
R_{2 n}(0) & =\Phi_{n}(0), \\
R_{2 n+1}(0) & =0, \quad n \neq N, \\
R_{2 N+1}(0) & =\gamma, \quad|\gamma| \neq 1 .
\end{aligned}
$$

This constitutes a finite perturbation (see [9]) of a symmetrized sequence (see [6,7]) corresponding to the reflection parameters associated with $\left(\Phi_{n}(0)\right)$. The framework of this problem is the analysis of some transformations of measures supported on the unit circle $($ see $[8,12])$ as a natural extension of the sieved process in the real line.

The aim of our contribution is the analysis of necessary and sufficient conditions in order that the sequence $\left(\Psi_{n}\right)$ defined in (3) be a MOPS.

\section{ORTHOGONALITY OF THE SEQUENCE $\left(\Psi_{n}\right)$}

Notice that if the sequence $\left(\Psi_{n}\right)$ introduced in (3) is an MOPS then the forward Szego" recurrence relation means

$$
\Psi_{n}(z)=z \Psi_{n}{ }_{1}(z)+\Psi_{n}(0) \Psi_{n}^{*}{ }_{1}(z) .
$$

Thus, we will consider three different cases for (3) taking into account the congruence modulus 3 for the degrees of the polynomials.

From $\Psi_{3 n}(z)=z \Psi_{3 n}{ }_{1}(z)+\Psi_{3 n}(0) \Psi_{3 n}^{*}{ }_{1}(z)$, taking into account (3) and (1), the identification of the polynomial components with the same cubic parity yields

$$
\left\{\begin{array}{l}
A_{n} \quad 1,1(z)=C_{n} \quad 1,1(z)+\Phi_{n}(0) C_{n}^{*} \quad 1,2(z), \\
A_{n} \quad{ }_{1,2}(z)=C_{n} \quad 1,2(z)+\Phi_{n}(0) C_{n}^{*} \quad 1,1 \\
{ }_{1,1}(z),
\end{array}\right.
$$

for every $n \geq 1$. Notice that $C_{n}^{*}(z):=z^{n} \bar{C}_{n}\left(z^{1}\right)$ despite the fact that $\operatorname{deg} C_{n} \leq n$.

In the same way, from $\Psi_{3 n+1}(z)=z \Psi_{3 n}(z)+\Psi_{3 n+1}(0) \Psi_{3 n}^{*}(z)$ we have

$$
\left\{\begin{array}{l}
B_{n, 1}(z)=z A_{n} \quad 1,2(z)+B_{n, 1}(0) \Phi_{n}^{*}(z), \\
B_{n} \quad{ }_{1,2}(z)=A_{n} \quad 1,1(z)+B_{n, 1}(0) A_{n}^{*} \quad(1,1 \\
B_{n, 1}(0) A_{n \quad 1,2}^{*}(z)=0,
\end{array}\right.
$$

Notice that $\Psi_{1}(z)=z+B_{0,1}(z)$, i.e., $B_{0,1}(z)=\Psi_{1}(0)$.

Finally $\Psi_{3 n+2}(z)=z \Psi_{3 n+1}(z)+\Psi_{3 n+2}(0) \Psi_{3 n+1}^{*}(z)$ yields

$$
\left\{\begin{array}{l}
C_{n, 1}(z)=z B_{n} \quad 1,2(z)+C_{n, 1}(0) \Phi_{n}^{*}(z), \\
C_{n, 2}(z)=B_{n, 1}(z)+C_{n, 1}(0) B_{n, 1}^{*}(z), \\
C_{n, 1}(0) B_{n}^{*} \quad 1,2(z)=0,
\end{array}\right.
$$

for every $n \geq 0$. 
Thus, we get

THEOREM 4 Let $\left(\Psi_{n}\right)$ be the polynomial sequence defined in (3), where $\left(\Phi_{n}\right)$ is a MOPS. $\left(\Psi_{n}\right)$ is a MOPS if and only if (5), (6) and (7) hold together with the fact that $\left|B_{n, 1}(0)\right| \neq 1$ and $\left|C_{n, 1}(0)\right| \neq 1$ for every $n \geq 1$.

In the next Proposition, we deduce a sufficient condition in order $\left(\Psi_{n}\right)$ be a MOPS, more simple than the above one. We use the following notation:

$$
\mathbb{A}_{n}(z)=\left(\begin{array}{l}
A_{n, 1}(z) \\
A_{n, 2}(z)
\end{array}\right), \quad \mathbb{A}_{n}^{*}(z)=\left(\begin{array}{l}
A_{n, 1}^{*}(z) \\
A_{n, 2}^{*}(z)
\end{array}\right), \quad J=\left(\begin{array}{ll}
0 & 1 \\
1 & 0
\end{array}\right),
$$

Proposition 5 Assume that $\left(\Phi_{n}\right)$ is a MOPS and consider the sequence of monic polynomials $\left(\Psi_{n}\right)$ defined in (3). If $\left(\Psi_{n}\right)$ is a MOPS, then there exists at most one positive integer number $N$ such that $B_{N, 1}(0) C_{N, 1}(0) \neq 0$. For such a $N, B_{n, 1}(0)=C_{n, 1}(0)=0$ for every $n \neq N$. The matrix sequence $\left(\mathbb{A}_{n}\right)$ satisfies the recurrence relation

$$
\mathbb{A}_{n}(z)=z \mathbb{A}_{n} 1(z)+\Phi_{n+1}(0) J \mathbb{A}_{n}^{*}{ }_{1}(z), \quad n \geq N+1
$$

and initial conditions

$$
\begin{aligned}
\mathbb{A}_{N}(z)= & \Phi_{N+1}(0)\left(\begin{array}{c}
\overline{B_{N, 1}(0)} \\
\overline{C_{N, 1}(0)}
\end{array}\right) \Phi_{N}(z) \\
& +\left(\begin{array}{c}
C_{N, 1}(0)+\Phi_{N+1}(0) \overline{C_{N, 1}(0)} B_{N, 1}(0) \\
B_{N, 1}(0)+C_{N, 1}(0) \overline{B_{N, 1}(0)}
\end{array}\right) \Phi_{N}^{*}(z) .
\end{aligned}
$$

Proof If for some $m \in \mathbb{N} B_{m, 1}(0) C_{m, 1}(0) \neq 0$, then from (6) $)_{3}$ we get $A_{m} \quad 1,2 \equiv 0$. Thus (5) 2 becomes $C_{m} \quad 1,2(z)=-\Phi_{m}(0) C_{m}^{*}{ }_{1,1}(z)$ and then $(5)_{1}$ leads to

$$
A_{m}{ }_{1,1}(z)=\left(1-\left|\Phi_{n}(0)\right|^{2}\right) C_{m} \quad 1,1(z) .
$$

From $(7)_{3}$ we get $B_{m} \quad 1,2 \equiv 0$. Thus in $(6)_{2}$

$$
A_{m}{ }_{1,1}(z)=-B_{m, 1}(0) A_{m}^{*}{ }_{1,1}(z),
$$

i.e.,

$$
\left(1-\left|B_{m, 1}(0)\right|^{2}\right) A_{m} \quad 1,1(z)=0 .
$$

Since $\left|B_{m, 1}(0)\right| \neq 1$, then $A_{m} \quad 1,1 \equiv 0$. Thus, $C_{m} \quad 1,1 \equiv 0 \equiv C_{m} \quad 1,2$ and so we get

$$
\Psi_{3 m} \quad 1(z)=z^{2} \Phi_{m} \quad 1\left(z^{3}\right) .
$$

Thus from (4)

$$
\Psi_{3 m} \quad 2(z)=z \Phi_{m} \quad 1\left(z^{3}\right)
$$

as well as

$$
\Psi_{3 m} 3(z)=\Phi_{m} 1\left(z^{3}\right) .
$$


In other words

$$
A_{m} 2,1 \equiv A_{m} 2,2 \equiv B_{m} \quad 1,1 \equiv B_{m} 2,2 \equiv 0
$$

But from (5),

$$
C_{m 2,1}=C_{m} 2,2=0
$$

i.e.,

$$
\begin{aligned}
& \Psi_{3 m}{ }_{4}(z)=z^{2} \Phi_{m} \quad 2\left(z^{3}\right), \\
& \Psi_{3 m}{ }_{5}(z)=z \Phi_{m} 2\left(z^{3}\right), \\
& \Psi_{3 m}{ }_{6}(z)=\Phi_{m}{ }_{2}\left(z^{3}\right) .
\end{aligned}
$$

Then, $B_{n, 1}(0)=C_{n, 1}(0)=0 \forall n<m$.

Thus if there exist two positive integer values $N, N^{\prime}\left(N<N^{\prime}\right)$ such that $B_{N, 1}(0) C_{N, 1}(0) \neq 0, \quad B_{N^{\prime}, 1}(0) C_{N^{\prime}, 1}(0) \neq 0$, we get a contradiction.

Finally, from $(6)_{1}$

$$
B_{m, 1}(z)=B_{m, 1}(0) \Phi_{m}^{*}(z)
$$

and from $(7)_{1}$ and $(7)_{2}$

$$
\left\{\begin{array}{l}
C_{m, 1}(z)=C_{m, 1}(0) \Phi_{m}^{*}(z) \\
C_{m, 2}(z)=B_{m, 1}(0) \Phi_{m}^{*}(z)+C_{m, 1}(0) \overline{B_{m, 1}(0)} \Phi_{m}(z) .
\end{array}\right.
$$

In other words, we have the explicit expressions for the elements $\left\{\Psi_{3 m}, \Psi_{3 m+1}, \Psi_{3 m+2}\right\}$. Indeed,

$$
\begin{aligned}
\Psi_{3 m}(z) & =\Phi_{m}\left(z^{3}\right), \\
\Psi_{3 m+1}(z) & =z \Phi_{m}\left(z^{3}\right)+B_{m, 1}(0) \Phi_{m}^{*}\left(z^{3}\right), \\
\Psi_{3 m+2}(z) & =\left[z+C_{m, 1}(0) \overline{B_{m, 1}(0)}\right] z \Phi_{m}\left(z^{3}\right)+\left[z B_{m, 1}(0)+C_{m, 1}(0)\right] \Phi_{m}^{*}\left(z^{3}\right) .
\end{aligned}
$$

If we assume the existence of one $N \in \mathbb{N}$ such that the above condition holds, we can analyze three cases. In what follows, for the sake of simplicity we will write $\beta=B_{N, 1}(0)$ and $\gamma=C_{N, 1}(0)$.

C.1 $B_{N+1,1}(0)=0, \quad C_{N+1,1}(0) \neq 0$.

Thus from (6)

$$
\begin{gathered}
B_{N+1,1}(z)=z A_{N, 2}(z), \\
B_{N, 2}(z)=A_{N, 1}(z) .
\end{gathered}
$$

But from $(7)_{3}, B_{N, 2} \equiv 0$, i.e., $A_{N, 1} \equiv 0$.

On the other hand, in (7)

$$
\begin{aligned}
& C_{N+1,1}(z)=C_{N+1,1}(0) \Phi_{N+1}^{*}(z), \\
& C_{N+1,2}(z)=B_{N+1,1}(z)+C_{N+1,1}(0) B_{N+1,1}^{*}(z) .
\end{aligned}
$$


Finally, in (5), $C_{N, 1}(z)=-\Phi_{N+1}(0) C_{N, 2}^{*}(z)$.

Taking into account the expressions (10) we get

$$
\gamma \Phi_{N}^{*}(z)=-\Phi_{N+1}(0)\left(\bar{\beta} \Phi_{N}(z)+\beta \bar{\gamma} \Phi_{N}^{*}(z)\right) .
$$

This means

$$
-\Phi_{N+1}(0) \bar{\beta} \Phi_{N}(z)=\left(\gamma+\beta \bar{\gamma} \Phi_{N+1}(0)\right) \Phi_{N}^{*}(z) .
$$

Then, from Proposition 3,

$$
0=-\Phi_{N+1}(0) \bar{\beta}=\gamma+\beta \bar{\gamma} \Phi_{N+1}(0) .
$$

Thus $\gamma=C_{N, 1}(0)=0$, a contradiction with our initial hypothesis.

C.2 $B_{N+1,1}(0) \neq 0, \quad C_{N+1,1}(0)=0$.

In an analog way as in the above case, we get a contradiction.

C.3 $B_{N+1,1}(0)=0, \quad C_{N+1,1}(0)=0$.

These conditions mean that

$$
\Psi_{3 N+4}(0)=0
$$

and then,

$$
\Psi_{3 N+4}(z)={ }_{z} \Psi_{3 N+3}(z) .
$$

Now, keeping in mind that

$$
\Psi_{3 N+5}(0)=0
$$

we can write

$$
\Psi_{3 N+5}(z)=z \Psi_{3 N+4}(z)=z^{2} \Psi_{3 N+3}(z) .
$$

This means that the associated reflection parameters are $\left\{\Phi_{N+1}(0), 0,0\right\}$.

Finally, the explicit expression for $\Psi_{3 N+3}(z)$ is

$$
\begin{aligned}
\Psi_{3 N+3}(z)= & \Phi_{N+1}\left(z^{3}\right)+z A_{N, 1}\left(z^{3}\right)+z^{2} A_{N, 2}\left(z^{3}\right) \\
= & z\left(z^{2}+\bar{\beta} \gamma z+\bar{\beta} \Phi_{N+1}(0)\right) \Phi_{N}\left(z^{3}\right) \\
& +\left[\left(\beta+\bar{\gamma} \Phi_{N+1}(0)\right) z^{2}+\left(\gamma+\beta \bar{\gamma} \Phi_{N+1}(0)\right) z+\beta \bar{\gamma}+\Phi_{N+1}(0)\right] \Phi_{N}^{*}\left(z^{3}\right) .
\end{aligned}
$$

Now, for the following term, we will consider another two cases.

$$
\begin{array}{lll}
\text { D.1 } & B_{N+1,1}(0)=0, & C_{N+1,1}(0)=0, \\
& B_{N+2,1}(0) \neq 0, & C_{N+2,1}(0)=0 .
\end{array}
$$

Since $B_{N+1,1}(0)=0$, from $(6)_{1}$ and $(6)_{2}$ we have

$$
\begin{gathered}
B_{N+1,1}(z)=z A_{N, 2}(z), \\
B_{N, 2}(z)=A_{N, 1}(z) .
\end{gathered}
$$


In the same way, since $C_{N+1,1}(0)=0,(7)_{1}$ and $(7)_{2}$ lead to

$$
\begin{aligned}
& C_{N+1,1}(z)=z B_{N, 2}(z)=z A_{N, 1}(z), \\
& C_{N+1,2}(z)=B_{N+1,1}(z)=z A_{N, 2}(z),
\end{aligned}
$$

and from (5), we obtain

$$
\begin{aligned}
& A_{N, 1}(z)=C_{N, 1}(z)+\Phi_{N+1}(0) C_{N, 2}^{*}(z), \\
& A_{N, 2}(z)=C_{N, 2}(z)+\Phi_{N+1}(0) C_{N, 1}^{*}(z) .
\end{aligned}
$$

Now, if we use the expressions for $C_{N, 1}(z)$ and $C_{N, 2}(z)$ as in (10) we can deduce

$$
A_{N, 1}(z)=\gamma \Phi_{N}^{*}(z)+\bar{\beta} \gamma \Phi_{N}(z)+\Phi_{N+1}(0)\left[\bar{\beta} \Phi_{N}(z)+\beta \bar{\gamma} \Phi_{N}^{*}(z)\right]
$$

On the other hand, from $(6)_{3}$ we have $A_{N+1,2} \equiv 0$. Now, $(6)_{1}$ and $(6)_{2}$ become

$$
\left\{\begin{array}{l}
B_{N+2,1}(z)=B_{N+2,1}(0) \Phi_{N+2}^{*}(z) \\
B_{N+1,2}(z)=A_{N+1,1}(z)+B_{N+2,1}(0) A_{N+1,1}^{*}(z)
\end{array}\right.
$$

and $(7)_{1},(7)_{2}$ lead to

$$
\begin{aligned}
& C_{N+2,1}(z)=z B_{N+1,2}(z), \\
& C_{N+2,2}(z)=B_{N+2,1}(z) .
\end{aligned}
$$

Keeping in mind (5), it follows that

$$
\begin{array}{r}
A_{N+1,1}(z)=z A_{N, 1}(z)+\Phi_{N+2}(0) A_{N, 2}^{*}(z), \\
0=A_{N+1,2}(z)=z A_{N, 2}(z)+\Phi_{N+2}(0) A_{N, 1}^{*}(z),
\end{array}
$$

and thus

$$
A_{N+1,1}(z)=z\left(1-\left|\Phi_{N+2}(0)\right|^{2}\right) A_{N, 1}(z),
$$

which we can written in the following way

$$
\begin{aligned}
0= & z\left[C_{N, 2}(z)+\Phi_{N+1}(0) C_{N, 1}^{*}(z)\right] \\
& +\Phi_{N+2}(0)\left[C_{N, 1}^{*}(z)+\overline{\Phi_{N+1}(0)} C_{N, 2}(z)\right] \\
= & \beta\left(z+\overline{\Phi_{N+1}(0)} \Phi_{N+2}(0)\right) \Phi_{N}^{*}(z) \\
& +\left[\bar{\gamma}\left(\Phi_{N+1}(0) z+\Phi_{N+2}(0)\right)+\bar{\beta} \gamma\right] \Phi_{N}(z)
\end{aligned}
$$

and, taking into account Proposition 3, we get $\beta=B_{N, 1}(0)=0$ which is a contradiction. 

D.2 $B_{N+1,1}(0)=0, \quad C_{N+1,1}(0)=0$, $B_{N+2,1}(0)=0, \quad C_{N+2,1}(0) \neq 0$.

A similar procedure yields a contradiction.

Thus, we can suppose that

$$
\begin{aligned}
\beta & =B_{N, 1}(0) \neq 0, \quad \gamma=C_{N, 1}(0) \neq 0, \\
B_{N+k, 1}(0) & =0, \quad C_{N+k, 1}(0)=0, \quad k=1, \ldots, n, \quad n \in \mathbb{N}
\end{aligned}
$$

and in a similar way as in the above cases using an induction process, we obtain

$$
B_{N+n+1,1}(0)=0, \quad C_{N+n+1,1}(0)=0, \quad n \in \mathbb{N} .
$$

As a conclusion,

$$
B_{n, 1}(0)=C_{n, 1}(0)=0, \quad \forall n \geq N+1 .
$$

In other words,

$$
\begin{aligned}
& \left\{\begin{array}{l}
B_{n, 1}(z)=z A_{n} \quad 1,2(z), \\
B_{n} \quad 1,2(z)=A_{n} \quad 1,1(z),
\end{array}\right. \\
& \left\{\begin{array}{l}
C_{n, 1}(z)=z B_{n} \quad 1,2(z), \\
C_{n, 2}(z)=B_{n, 1}(z)
\end{array}\right.
\end{aligned}
$$

for $n \geq N+1$. Thus

$$
\begin{aligned}
& \Psi_{3 n+1}(z)=z \Psi_{3 n}(z), \quad n>N, \\
& \Psi_{3 n+2}(z)=z^{2} \Psi_{3 n}(z), \quad n>N, \\
& \Psi_{3 n+3}(z)=\Phi_{n+1}\left(z^{3}\right)+z A_{n, 1}\left(z^{3}\right)+z^{2} A_{n, 2}\left(z^{3}\right), \quad n \geq N,
\end{aligned}
$$

and

$$
\mathbb{A}_{n}(z)=z \mathbb{A}_{n}{ }_{1}(z)+\Phi_{n+1}(0) J \mathbb{A}_{n}^{*}{ }_{1}(z)
$$

for $n \geq N+1$ with the initial conditions (8).

The next step will be an alternative way to deduce an explicit expression for the sequence $\left(\Psi_{n}\right)$ in terms of the sequences $\left(\Phi_{n}\right)$ and $\left(\Omega_{n}\right)$, where $\left(\Omega_{n}\right)$ is the MOPS of the second kind for $\left(\Phi_{n}\right)$ (see $\left.[5,9]\right)$.

Taking into account the above results, the sequence $\left(\Psi_{n}\right)$ is a finite perturbation of $\left(\widetilde{\Psi}_{n}\right)$ at level $3 N+2$, where $\left(\widetilde{\Psi}_{n}\right)$ is the MOPS obtained from the sequence $\left(\Psi_{n}\right)$ introducing two zeros between two consecutive terms of the corresponding sequence of 
reflection parameters. By means of $\Lambda_{n}$ and $\widetilde{\Lambda}_{n}$ we denote the polynomials of the second kind of $\Psi_{n}$ and $\widetilde{\Psi}_{n}$ respectively. Using the procedure described in [3] we get

$$
\left(\begin{array}{c}
\Psi_{3 N+2+k}(z) \\
\Lambda_{3 N+2+k}(z)
\end{array}\right)=\frac{1}{2 z^{3 N+2} e_{N}}\left(\begin{array}{cc}
R(z) & S(z) \\
U(z) & V(z)
\end{array}\right)\left(\begin{array}{c}
\widetilde{\Psi}_{3 N+2+k}(z) \\
\widetilde{\Lambda}_{3 N+2+k}(z)
\end{array}\right)
$$

where

$$
\left(\begin{array}{cc}
R(z) & S(z) \\
U(z) & V(z)
\end{array}\right)=\left(\begin{array}{cc}
\Phi_{N}^{*}\left(z^{3}\right) & \Phi_{N}\left(z^{3}\right) \\
-\Omega_{N}^{*}\left(z^{3}\right) & \Omega_{N}\left(z^{3}\right)
\end{array}\right)\left(\begin{array}{cc}
p(z) & q(z) \\
z q^{*}(z) & z p^{*}(z)
\end{array}\right)\left(\begin{array}{cc}
z^{2} \Omega_{N}\left(z^{3}\right) & -z^{2} \Phi_{N}\left(z^{3}\right) \\
\Omega_{N}^{*}\left(z^{3}\right) & \Phi_{N}^{*}\left(z^{3}\right)
\end{array}\right),
$$

with

$$
\begin{aligned}
& p(z)=\beta \bar{\gamma} z+1, \\
& q(z)=\beta z+\gamma .
\end{aligned}
$$

Notice that $R, S, U, V$ are self-reciprocal polynomials (see [9]).

In the same way, if we denote by $\widetilde{F}$ the Carathéodory function associated with the MOPS $\left(\widetilde{\Psi}_{n}\right)$, then it is known ([6]) that $\widetilde{F}(z)=F\left(z^{3}\right)$. Thus

Proposition 6 For the Carathéodory function $G$ associated with the MOPS $\left(\Psi_{n}\right)$ we get

$$
G(z)=\frac{U(z) F\left(z^{3}\right)+V(z)}{R(z) F\left(z^{3}\right)+S(z)}, \quad z \in \mathbb{D}=\{|z|<1\} .
$$

Proof For $n \geq 3 N+2$ we can write

$$
\frac{\Lambda_{n}^{*}(z)}{\Psi_{n}^{*}(z)}=\frac{U(z) \tilde{\Psi}_{n}^{*}(z)+V(z) \widetilde{\Lambda}_{n}^{*}(z)}{R(z) \widetilde{\Psi}_{n}^{*}(z)+S(z) \widetilde{\Lambda}_{n}^{*}(z)}
$$

Then (see [10])

$$
G(z)=\lim _{n} \frac{\Lambda_{n}^{*}(z)}{\Psi_{n}^{*}(z)}=\frac{U(z) F\left(z^{3}\right)+V(z)}{R(z) F\left(z^{3}\right)+S(z)}, \quad z \in \mathbb{D} .
$$

Remember that in the positive definite case the measure $d \mu$ belongs to the Szego class when $\left(\Phi_{n}(0)\right) \in l_{2}$. In this case we can define the Szegö function as

$$
D(z ; d \mu)=\exp \left\{\frac{1}{4 \pi} \int_{\pi}^{\pi} \frac{1+z e^{i \theta}}{1-z e^{i \theta}} \log \mu^{\prime}(\theta) d \theta\right\}, \quad z \in \mathbb{D},
$$

(see [13]) and furthermore

$$
D(z ; d \mu)=\lim _{n} \frac{\kappa_{n}}{\Phi_{n}^{*}(z)}
$$

locally uniformly in $\mathbb{D}$, where $\kappa_{n}=\left\|\Phi_{n}\right\|^{2}$. Now, it is easy to obtain the Szego function associated with the polynomials $\Psi_{n}$. 
Proposition 7 Let $d \mu$ be a positive Borel measure supported on $\mathbb{T}$ which belongs to the Szego class. Let $\left(\Psi_{n}\right)$ be the MOPS introduced as above and denote $B_{N, 1}(0)=\beta$, $C_{N, 1}(0)=\gamma$. If $\beta, \gamma \in \mathbb{D}$, then the measure $d v$ of orthogonality for $\left(\Psi_{n}\right)$ belongs to the Szego class and the corresponding Szego function is given by

$$
D(z ; d \nu)=\left[\left(1-|\beta|^{2}\right)\left(1-|\gamma|^{2}\right)\right]^{1 / 2} \frac{D\left(z^{3}, d \mu\right)}{R(z) F\left(z^{3}\right)+S(z)}, \quad z \in \mathbb{D} .
$$

As a consequence,

$$
v^{\prime}(\theta)=\left[\left(1-|\beta|^{2}\right)\left(1-|\gamma|^{2}\right)\right]^{1 / 2} \frac{\mu^{\prime}(3 \theta)}{\left|R\left(e^{i \theta}\right) F\left(e^{3 i \theta}\right)+S\left(e^{i \theta}\right)\right|^{2}},
$$

where $\mu^{\prime}$ and $v^{\prime}$ denote, respectively, the Radon-Nikodym derivatives of $\mu$ and $v$ with respect to the Lebesgue measure.

\section{SOME EXAMPLES}

1. We illustrate the preceding results with some examples. First, we consider the sequence of reflection parameters given by

$$
\Phi_{n}(0)=\frac{1}{n+1}, \quad n \in \mathbb{N} \cup\{0\} .
$$

It is very well known that the corresponding sequence of orthogonal polynomials $\left(\Phi_{n}\right)$ is

$$
\Phi_{n}(z)=\frac{1}{n+1} \sum_{k=0}^{n}(k+1) z^{k}=\frac{1}{n+1} \frac{(n+1) z^{n+2}-(n+2) z^{n+1}+1}{(1-z)^{2}} .
$$

For the polynomials of second kind we get

$$
\Omega_{n}(z)=\frac{1}{n+1} \frac{(n+1) z^{n+1}-(n+2) z^{n}+1}{(z-1)} .
$$

Besides, the corresponding orthogonality measure $d \mu$ constitutes a modification of the Lebesgue measure. More precisely,

$$
d \mu(\theta)=\frac{1}{4 \pi}\left|e^{i \theta}-1\right|^{2} d \theta
$$

and we can obtain the sequence $\left\{u_{n}\right\}_{n \geq 0}$ of the moments for the measure $d \mu$. In fact

$$
\begin{aligned}
u_{k} & =\frac{1}{4 \pi} \int_{0}^{2 \pi} e^{i k \theta}\left|e^{i \theta}-1\right|^{2} d \theta \\
& \left.=\frac{1}{4 \pi} \int_{0}^{2 \pi}\left(2 e^{i k \theta}-e^{i(k+1) \theta}-e^{i(k} 1\right) \theta\right) d \theta=0, \quad k \in \mathbb{Z},
\end{aligned}
$$


up to $k \in\{0,-1,1\}$. Notice that

$$
u_{0}=1, \quad u_{1}=u_{1}=-\frac{1}{2}
$$

Thus, the corresponding Carathéodory function is

$$
F(z)=1-z, \quad z \in \mathbb{D} \text {. }
$$

In order to obtain the Carathéodory function related to the sequence $\left(\Psi_{n}\right)_{n \in \mathbb{N}}$, taking into account the expression for the sequences $\Phi_{n}, \Omega_{n}$, their corresponding reciprocal polynomials, and keeping the notation of the previous sections, now we can write

$$
\begin{gathered}
\left(\begin{array}{cc}
R(z) & S(z) \\
U(z) & V(z)
\end{array}\right)=\frac{1}{\left(z^{3}-1\right)}\left(\begin{array}{cc}
r(z) & s(z) \\
u(z) & v(z)
\end{array}\right) \\
\left(\begin{array}{c}
z^{2}\left(z^{3}-1\right)\left((N+1) z^{3 N}-\sum_{k=0}^{N} z^{3 k}\right)-z^{2}\left((N+1) z^{3 N+3}-\sum_{k=0}^{N} z^{3 k}\right) \\
\left(z^{3}-1\right)\left(\sum_{k=0}^{N} z^{3 k}-(N+1)\right)
\end{array}\right)
\end{gathered}
$$

where $r, s$ are polynomials of degree exactly $3 N+2$ with leading coefficient $\bar{\gamma}$ and $u, v$ are monic polynomials of degree $3 N+2$. More precisely

$$
\begin{aligned}
& \left(\begin{array}{cc}
r(z) & s(z) \\
u(z) & v(z)
\end{array}\right)=\frac{1}{\left(z^{3}-1\right)(N+1)}\left(\begin{array}{cc}
\beta \bar{\gamma} z+1 & \bar{\gamma} z^{2}+\bar{\beta} z \\
\beta z+\gamma & z^{2}+\bar{\beta} \gamma z
\end{array}\right) \\
& \left.\left(\begin{array}{cc}
\sum_{k=0}^{N} z^{3 k}-(N+1) & -\left(z^{3}-1\right)\left(\sum_{k=0}^{N} z^{3 k}-(N+1)\right.
\end{array}\right)\right)
\end{aligned}
$$

As a consequence of Proposition 6 we deduce the corresponding expression for the Carathéodory function

$$
G(z)=\frac{\left(z^{3}-1\right) U(z)-V(z)}{\left(z^{3}-1\right) R(z)-S(z)}, \quad z \in \mathbb{D}
$$

and from Proposition 7 it follows that

$$
v^{\prime}(\theta)=\left[\left(1-|\beta|^{2}\right)\left(1-|\gamma|^{2}\right)\right]^{1 / 2} \frac{1-\cos 3 \theta}{\left|R\left(e^{i \theta}\right)\left(1-e^{3 i \theta}\right)+S\left(e^{i \theta}\right)\right|^{2}} .
$$


2. We will consider another modification of the Lebesgue measure. This is given by means of the Poisson kernel $P_{r}(\theta)$, i.e.,

$$
d \mu(\theta)=P_{|a|}(k \theta-t) d \theta=\frac{1-|a|^{2}}{\left|a+e^{i k \theta}\right|^{2}} d \theta
$$

where $a=|a| e^{i t} \in \mathbb{D}$ and $k \in \mathbb{N}$ fixed.

The corresponding sequence of orthogonal polynomials is called a Bernstein-Szego" polynomial sequence, i.e., their reflection parameters are

$$
\begin{aligned}
& \Phi_{n}(0)=0, \quad n \in \mathbb{N}, \quad n \neq k, \\
& \Phi_{k}(0)=a .
\end{aligned}
$$

Keeping in mind the forward recurrence relation, for the sequence $\left(\Phi_{n}\right)_{n \geq 0}$ we obtain

$$
\begin{array}{ll}
\Phi_{n}(z)=z^{n}, & n<k \\
\Phi_{n}(z)=z^{n k}\left(z^{k}+a\right), & n \geq k .
\end{array}
$$

Thus, the Carathéodory function is a rational function. More precisely,

$$
F(z)=\frac{-\bar{a} z^{k}+1}{\bar{a} z^{k}+1}
$$

If we make a perturbation at level $3 N+2$ with $N \geq k$ for the polynomials $\Psi_{n}$ and $\Lambda_{n}$ we get

$$
\begin{aligned}
& \Psi_{3 N+2}^{*}(z)=(\bar{\gamma} \bar{\beta} z+1) \widetilde{\Psi}_{3 N}^{*}(z)+(\bar{\gamma} z+\bar{\beta}) z \widetilde{\Psi}_{3 N}(z), \\
& \Lambda_{3 N+2}^{*}(z)=(\bar{\gamma} \bar{\beta} z+1) \widetilde{\Lambda}_{3 N}^{*}(z)+(-\bar{\gamma} z+\bar{\beta}) z \widetilde{\Lambda}_{3 N}(z) .
\end{aligned}
$$

As a consequence

$$
\begin{aligned}
F(z) & =\frac{\Lambda_{3 N+2}^{*}(z)}{\Psi_{3 N+2}^{*}(z)}=\frac{(\bar{\gamma} \bar{\beta} z+1) \Omega_{k}^{*}\left(z^{3}\right)+(-\bar{\gamma} z+\bar{\beta}) z^{3(N \quad k)+1} \Omega_{k}\left(z^{3}\right)}{(\bar{\gamma} \bar{\beta} z+1) \Phi_{k}^{*}\left(z^{3}\right)+(\bar{\gamma} z+\bar{\beta}) z^{3(N k)+1} \Phi_{k}\left(z^{3}\right)} \\
& =\frac{(\bar{\gamma} \bar{\beta} z+1)\left(-\bar{a} z^{3 k}+1\right)+(-\bar{\gamma} z+\bar{\beta}) z^{3(N k)+1}\left(z^{3 k}-a\right)}{(\bar{\gamma} \bar{\beta} z+1)\left(\bar{a} z^{3 k}+1\right)+(\bar{\gamma} z+\bar{\beta}) z^{3(N k)+1}\left(z^{3 k}+a\right)}
\end{aligned}
$$

The Szego function for the modified sequence is (see[3]).

$$
D(z ; d \mu)=\frac{\left(1-|\gamma|^{2}\right)^{1 / 2}\left(1-|\beta|^{2}\right)^{1 / 2} \kappa_{k}}{(\bar{\gamma} \bar{\beta} z+1)\left(\bar{a} z^{3 k}+1\right)+(\bar{\gamma} z+\bar{\beta}) z^{3(N k)+1}\left(z^{3 k}+1\right)}, \quad z \in \mathbb{D} .
$$




\section{Acknowledgements}

The work of the first author was supported by Dirección General de Investigación (Ministerio de Ciencia y Tecnología) of Spain under grant BFM 2000-0206-C04-03. The work of the second author was supported by Dirección General de Enseñanza Superior (DGES) of Spain under grant PB 98-1615. The work of the third author was supported by Dirección General de Investigación (Ministerio de Ciencia y Tecnología) of Spain under grant BFM 2000-0206-C04-01 and INTAS project 2000-272.

\section{References}

[1] A. Atzmon (1998). n Orthonormal operator polynomials. In: Gohberg (Ed.), Operator Theory: Advances and Applications, Vol. 34(I), pp. 47 63. Birkhäuser Basel.

[2] M. Bakonyi and T. Constantinescu (1992). Schur's algorithm and several applications. Pitman Research Notes in Mathematics, Series 261. Longman Scientific Technical, Essex, UK.

[3] M.J. Cantero, F. Marcellán and L. Moral (2001). A class of non symmetric orthogonal polynomials on the unit circle. J. Approx. Theory, 109, 3047.

[4] T. Erdelyi, J.S. Geronimo, P. Nevai and J. Zhang (1991). A simple proof of "Favard's Theorem" on the unit circle. Atti Sem. Mat. Fis. Univ. Modena, 29, 4146.

[5] Ya. L. Geronimus (1962). Polynomials orthogonal on a circle and their applications. Amer. Math. Soc. Trans., 3, 178.

[6] M.E.H. Ismail and X. Li (1992). On sieved orthogonal polynomials IX: orthogonality on the unit circle. Pacific. J. Math., 153, 289297.

[7] F. Marcellán and G. Sansigre (1991). Orthogonal polynomials on the unit circle: symmetrization and quadratic decomposition. J. Approx. Theory, 65, 109119.

[8] F. Marcellán and J.C. Petronilho (1999). Orthogonal polynomials and polynomial mappings on the unit circle. In: V.B. Priezzhev and V.P. Spiridonov (Eds.), Proceedings of the International Workshop on Self Similar Systems, pp. 316 326, JINR ES 99 38. Dubna.

[9] F. Peherstorfer (1996). A special class of polynomials orthogonal on the unit circle including the associ ated polynomials. Constr. Approx., 12, 161185.

[10] F. Peherstorfer and R. Steinbauer (1995). Characterization of general orthogonal polynomials with respect to a functional. J. Comput. Appl. Math., 65, 339355.

[11] F. Peherstorfer and R. Steinbauer (1994). Perturbation of orthogonal polynomials on the unit circle a survey. In: M. Alfaro et al. (Ed.), Proceedings Workshop on Orthogonal Polynomials on the Unit Circle, pp. 97 119. Universidad Carlos III de Madrid, Leganés.

[12] F. Peherstorfer and R. Steinbauer (1998). Transformation of polynomials orthogonal on the unit circle. Methods Appl. Anal., 5, 5580.

[13] G. Szego (1975). Orthogonal polynomials. Amer. Math. Soc. Colloq. Publ., 23, 4th Edn. Providence, Rhode Island.

[14] W. Van Assche (1997). Orthogonal polynomials in the complex plane and on the real line. Fields Institute Communications, 14, 211245. 